\title{
Delivering pharmacogenetic testing to the masses: an achievable goal?
}

\section{"The public will likely play a large role in driving the uptake of pharmacogenetic screening and influence the delivery models."}

\section{KEYWORDS: delivery models $=$ pharmacogenetic testing $\approx$ public health whole-genome sequencing}

Ten years following the completion of the draft sequence of the human genome, some have wondered if the effort was worth the cost and what happened to the promise of a medical revolution [101]. Admittedly, the exuberance and exhilaration of having a completely sequenced genome led to some overstretched visions of the future [1]. Pharmacogenetics (PGx) is one example of promising more than can be currently delivered with catchy slogans such as the 'right drug at the right dose for the right patient' $[2,3]$. Although pharmacogenetics may not be moving as quickly as anticipated or desired, primarily owing to the lack of evidence of clinical utility, the community has made great strides in understanding the genetic basis of long-observed differences in drug response [4] and PGx has become a pillar of the personalized medicine movement. However, PGx research has also confirmed that genetic variation does not typically account for the majority of variability observed in drug response and more work is needed to discover and identify the combinations of genetic and nongenetic factors to develop better predictors of drug response.

Nonetheless, there is still value in some current PGx tests and great potential for its future use, and many institutions are developing or piloting programs to assess delivery strategies for PGx testing. Most of these delivery models are based on a prescription-driven model (testing ordered at time of prescription), and to a lesser extent, a pre-emptive model for patients anticipated to need therapeutic treatment in the near future (e.g., at the time of hospital admission). However, neither the prescriptiondriven model nor the pre-emptive model being explored now has considered strategies to deliver PGx to the larger population. Inevitably, everyone will require some type of pharmacological treatment during their lifetime, most likely requiring multiple treatments for various conditions. Prescription medication use is increasing in children and at younger ages [5,6]. Slightly different from the pre-emptive models being explored now, PGx screening (a pre-emptive PGx test but in the absence of an impending illness or health crisis) could be considered as a type of preventive healthcare service that would enable access to PGx information for the general population. However, the current challenges of delivering preventive healthcare and relatively low patient follow through [7-9] do not portend successful implementation of PGx screening as part of preventive care. In particular, given the lack of public familiarity with PGx testing, it will be critical to ensure that patients understand the purpose of testing and the potential importance of the results to all medications prescribed to them, something not likely feasible given time constraints of an office visit and limited physician knowledge about PGx.

Thus, to encourage more widespread and routine use of testing and to address potential disparities in the provision of PGx testing due to differences in provider knowledge and practice settings as well as insurance policies, new approaches should be considered. This editorial describes some delivery models of PGx screening for consideration in nontraditional healthcare settings.

\section{Option 1: incorporate into wellness programs}

Many employers and insurance companies have developed wellness programs to promote health prevention, improve the welfare of employees or clients and reduce overall healthcare expenditures. Modifiable health risks such as weight, smoking and hypertension, factors

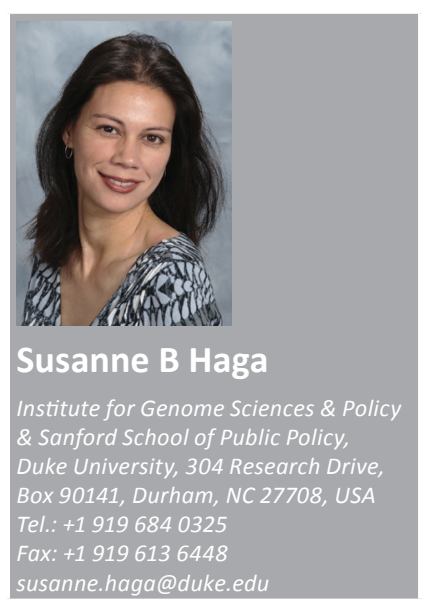

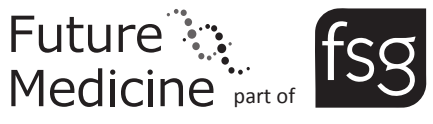


that account for a large proportion of employer health expenditures, can be improved through wellness programs [10]. Some programs may focus on individuals affected with chronic conditions who may particularly benefit from access to supportive services to improve health outcomes. Despite the popularity of such programs, their effectiveness remains unclear $[11,12]$, based on a limited number of careful evaluations conducted and due to differences between programs.

The addition of PGx screening to wellness programs represents a logical extension of current benefits such as medication therapy management and strategies to improve medication adherence. As with any wellness programs, employee/client participation is completely optional. Regardless of whether individuals decide to have a PGx screening test performed through the wellness program, they may benefit from learning about the potential impact of genes on drug response and ask their healthcare provider about testing the next time they are prescribed a new drug. If an individual decides to have PGx screening, the wellness program may also provide access to the appropriate services, such as a consultation with a genetic counselor and/or pharmacist, to ensure understanding and encourage sharing of the results with healthcare providers each time a new drug is prescribed. Alternatively, the individual can discuss the results directly with their healthcare provider. In addition to potentially improving medication dosing or selection, knowledge of one's PGx results may improve medication adherence by ameliorating concerns of risk of adverse response [13], both outcomes are likely to help reduce healthcare costs.

"The addition of pharmacogenetic screening
to wellness programs represents a logical
extension of current benefits such as
medication therapy management and
strategies to improve medication
adherence."

\section{Option 2: retail-based PGx clinics}

Provision of general clinical services at retailbased clinics (e.g., US-based MinuteClinic, TakeCare and LittleClinic) has been gaining in popularity [14-16], providing patients rapid and convenient access, of comparable quality to clinical care provided in a traditional practice-based setting but more affordable $[17,18]$. A 'pharmacogenetics' clinic could also be established, either as an additional or separate specialty service to current retail-based clinics, enabling patients to learn more about PGx screening from a health professional at the clinic, provide a DNA sample for testing, and discuss their results. The healthcare provider operating the PGx clinic could be a physician or nurse with training in genetics and/or pharmacy, a genetic counselor or pharmacist.

Alternatively, PGx screening could be added to the growing list of services provided by pharmacists including blood pressure monitoring, flu vaccinations, medication therapy management and patient counseling. Many of these nondispensing services have been demonstrated to improve patient outcomes $[19,20]$ and patients have embraced the role of pharmacists as providers of these additional services, even for children [21]. Indeed, pharmacists may be in a better position to provide PGx screening to high-risk patients taking multiple medications and counsel them about the significance of the results to their current medications. The results could then be added to their pharmacy profile and reviewed each time a new prescription is filled.

\section{"Indeed, pharmacists may be in a better position to provide pharmacogenetic screening to high-risk patients taking multiple medications and counsel them about the significance of the results to their current medications."}

Patients may be more likely to have PGx screening if recommended by their primary care provider [22]. Thus, a primary care office can suggest that patients undergo PGx screening and refer them to a PGx clinic affiliated with a retail-based clinic or pharmacy that can provide the necessary genetic or medication counseling. Furthermore, to promote awareness of PGx screening, pharmacy intake forms can add a question regarding the patient's PGx testing history and results, analogous to questions about drug allergies. This may lead to patients asking the pharmacist about PGx screening and having PGx testing through a PGx clinic.

\section{Option 3: whole-genome sequencing/whole-exome sequencing-based health risk assessment}

With the rapid utilization of whole-genome sequencing (WGS), whole-exome sequencing 
(WES) or array-based analysis of a few hundred genes associated with drug response [23,24], testing one gene at a time is quickly becoming an outdated approach. The cost of a series of single gene PGx tests can quickly exceed the costs of a more comprehensive genome-wide analysis or panel of genes. While WGS/WES is commonly used as a research tool, it is also being used in challenging clinical cases and cancer. Some have suggested that it be used as a health risk assessment [25] and several research groups recently have begun to investigate the potential utility of WGS/WES as part of the newborn screening program [102]. Several PGx genes are included in direct-to-consumer testing services, which analyze genetic variations across the genome on array-based platforms. For example, the company 23andMe includes 24 drug response results in its health risk assessment report [103].

"Since the number of prescribed medications is increasing in children and at younger ages, knowledge of a child's drug response profile may be of interest and of value to both parents and providers."

If WGS/WES is ordered clinically, in addition to the results that are considered relevant for the patient, there will also be incidental findings, or disease risk information unrelated to the primary clinical concern. There has been much debate about what incidental findings to disclose to the patient and in 2013, the American College of Medical Genetics and Genomics recommended that incidental results for 24 diseases be reported (no genes associated with drug response were included in that list) [26]. But patients may be interested in learning much more about the content of their genome, including genes linked to drug response [27]. In order to help patients make informed decisions about what types of information they may wish to learn about from their genome, it may be helpful to partition the genome into familiar disease groups or categories, providing patients with a 'menu' of options that they can then choose from. By carving the genome into smaller 'sections', patients may be less overwhelmed than having a list of hundreds of phenotypes presented, as is currently done with some direct-to-consumer companies. Drug response or PGx results can be included as one of the 'sections' available from the WGS/WES menu. This approach would be very similar to individual genetic testing panels for cardiology or cancer, though extracted from the WGS/WES data set.

Likewise, if WGS/WES is conducted as part of newborn screening, parents can be given the option to only access certain 'sections' of information most relevant for the child's immediate health and well-being and defer decisions to access other diseases to the child when they reach adulthood. Since the number of prescribed medications is increasing in children and at younger ages, knowledge of a child's drug response profile may be of interest and of value to both parents and providers.

\section{Discussion \& conclusion}

The current system of PGx test ordering based primarily on a prescription-driven model may severely limit the use of PGx information for the general public. While efforts are being made to educate healthcare providers, such as through clinical decision supports linked to electronic medical records, similar effort should be made to promote awareness of PGx testing among the public. The public will likely play a large role in driving the uptake of PGx screening and influence the delivery models. While public interest in PGx testing appears high [28], likely owing to personal experience with drug-related side effects and the trial-and-error approach to find a safe and effective drug and dose, the public's enthusiasm may be countered by concerns of genetics and privacy. The relatively low uptake of current screening recommendations in general suggests that PGx screening will also be of limited uptake if delivered in a traditional clinical setting. More strategies should be considered to deliver PGx testing to the general population, to develop tools to promote informed decision-making and reduce the cost of testing so that it is affordable for a large segment of the population. Until these goals are achieved, implementation of any of the delivery models discussed will likely meet with limited success.

Financial \& competing interests disclosure This work was supported by the US NIH (R01 GM081416). The author serves as a consultant to the Institute for Translational Medicine Institute at the INOVA Health Care Services. The author has no other relevant affiliations or financial involvement with any organization or entity with a financial interest in or financial conflict with the subject matter or materials discussed in the manuscript apart from those disclosed.

No writing assistance was utilized in the production of this manuscript. 


\section{References}

1 Collins F. Has the revolution arrived? Nature 464(7289), 674-675 (2010).

2 Nebert DW. Given the complexity of the human genome, can 'personalised medicine' or 'individualised drug therapy' ever be achieved? Hum. Genomics 3(4), 299-300 (2009).

3 Shah RR, Shah DR. Personalized medicine: is it a pharmacogenetic mirage? Br. J. Clin. Pharmacol. 74(4), 698-721 (2012).

4 Scott SA. Personalizing medicine with clinical pharmacogenetics. Genet. Med. 13(12), 987-995 (2011).

5 Chai G, Governale L, McMahon AW, Trinidad JP, Staffa J, Murphy D. Trends of outpatient prescription drug utilization in US children, 2002-2010. Pediatrics 130(1), 23-31 (2012).

6 Cox ER, Halloran DR, Homan SM, Welliver S, Mager DE. Trends in the prevalence of chronic medication use in children: 2002-2005. Pediatrics 122(5), e1053-e1061 (2008).

7 Stange KC, Flocke SA, Goodwin MA. Opportunistic preventive services delivery. Are time limitations and patient satisfaction barriers? J. Fam. Pract. 46(5), 419-424 (1998).

8 Yarnall KS, Pollak KI, Ostbye T, Krause KM, Michener JL. Primary care: is there enough time for prevention? Am. J. Public Health 93(4), 635-641 (2003).

9 Smith M, Bates DW, Bodenheimer T, Cleary PD. Why pharmacists belong in the medical home. Health Aff. (Millwood) 29(5), 906-913 (2010).

10 Anderson DR, Whitmer RW, Goetzel RZ et al. The relationship between modifiable health risks and group-level health care expenditures. Health Enhancement Research Organization (HERO) Research Committee. Am. J. Health Promot. 15(1), 45-52 (2000).

11 Baicker K, Cutler D, Song Z. Workplace wellness programs can generate savings. Health Aff. (Millwood) 29(2), 304-311 (2010).
12 Osilla KC, van Busum K, Schnyer C, Larkin JW, Eibner C, Mattke S. Systematic review of the impact of worksite wellness programs. Am. J. Manag. Care 18(2), e68-e81 (2012).

13 Haga SB, Lapointe NM. The potential impact of pharmacogenetic testing on medication adherence. Pharmacogenomics J. doi:10.1038/ tpj.2013.33 (2013) (Epub ahead of print).

14 Mehrotra A, Lave JR. Visits to retail clinics grew fourfold from 2007 to 2009, although their share of overall outpatient visits remains low. Health Aff. (Millwood) 31(9), 2123-2129 (2012).

15 Rudavsky R, Pollack CE, Mehrotra A. The geographic distribution, ownership, prices, and scope of practice at retail clinics. Ann. Intern. Med. 151(5), 315-320 (2009).

16 Uscher-Pines L, Harris KM, Burns RM, Mehrotra A. The growth of retail clinics in vaccination delivery in the U.S. Am. J. Prev. Med. 43(1), 63-66 (2012).

17 Mehrotra A, Liu H, Adams JL et al. Comparing costs and quality of care at retail clinics with that of other medical settings for 3 common illnesses. Ann. Intern. Med. 151(5), 321-328 (2009).

18 Reid RO, Ashwood JS, Friedberg MW, Weber ES, Setodji CM, Mehrotra A. Retail clinic visits and receipt of primary care. J. Gen. Intern. Med. 28(4), 504-512 (2013).

19 Nkansah N, Mostovetsky O, Yu C et al. Effect of outpatient pharmacists' nondispensing roles on patient outcomes and prescribing patterns. Cochrane Database Syst. Rev. 7, CD000336 (2010).

20 Taitel M, Cohen E, Duncan I, Pegus C. Pharmacists as providers: targeting pneumococcal vaccinations to high risk populations. Vaccine 29(45), 8073-8076 (2011).

21 Deshpande M, Schauer J, Mott DA, Young HN, Cory P. Parents' perceptions of pharmacists as providers of influenza vaccine to children. J. Am. Pharm. Assoc. 53(5), 488-495 (2013).

22 Costanza ME, Luckmann R, Stoddard AM et al. Using tailored telephone counseling to accelerate the adoption of colorectal cancer screening. Cancer Detect. Prev. 31(3), 191-198 (2007).
23 Burmester JK, Sedova M, Shapero MH, Mansfield E. DMET microarray technology for pharmacogenomics-based personalized medicine. Methods Mol. Biol. 632, 99-124 (2010).

24 Johnson JA, Burkley BM, Langaee TY, Clare-Salzler MJ, Klein TE, Altman RB. Implementing personalized medicine: development of a cost-effective customized pharmacogenetics genotyping array. Clin Pharmacol. Ther. 92(4), 437-439 (2012).

25 Patel CJ, Sivadas A, Tabassum R et al. Whole genome sequencing in support of wellness and health maintenance. Genome Med. 5(6), 58 (2013).

26 Green RC, Berg JS, Grody WW et al. ACMG recommendations for reporting of incidental findings in clinical exome and genome sequencing. Genet. Med. 15(7), 565-574 (2013).

27 Ashley EA, Butte AJ, Wheeler MT et al. Clinical assessment incorporating a personal genome. Lancet 375(9725), 1525-1535 (2010).

28 Haga SB, O’Daniel JM, Tindall GM, Lipkus IR, Agans R. Survey of US public attitudes toward pharmacogenetic testing. Pharmacogenomics J. 12(3), 197-204 (2012).

\section{Websites}

101 Connor S. Ten years ago today, it was revealed that the human genome had been decoded. A medical revolution beckoned. So what happened next? www.independent.co.uk/news/science/tenyears-ago-today-it-was-revealed-that-thehuman-genome-had-been-decoded-a-medicalrevolution-beckoned-so-what-happenednext-2011016.html (Accessed 11 October 2013)

102 NIH: NIH program explores the use of genomic sequencing in newborn healthcare. www.nih.gov/news/health/sep2013/nhgri-04. htm

(Accessed 11 October 2013)

103 23andMe.

www.23andme.com

(Accessed 11 October 2013) 